\title{
The Basics of Noise Detection and Filtering for Borehole Drilling Data
}

\author{
Meen-Wah Gui*
}

\author{
Department of Civil Engineering, National Taipei University of Technology, Taipei 106, Taiwan
}

\begin{abstract}
A series of borehole instrumented drilling tests have been performed at two separate sites in London. However, these data contain noise which makes interpretation difficult. A study was thus carried out to explore the possibility of using signal processing techniques to remove noise from the instrumented borehole drilling data. The study began by examining the most common methods used to detect noise in a signal. Three types of filter: moving average, median and Butterworth filters were compared. Filtering weight for moving average filter, filtering order for median filter, and cut-off frequency for Butterworth filter were then proposed for each of the drilling parameters. The effects of standardized and non-standardized drilling procedures on the drilling data were also demonstrated by using cross-correlation functions for groups of standardized and non-standardized tests.
\end{abstract}

\section{INTRODUCTION}

The concept of instrumented drilling has long been applied in the oil and gas industry, but it is still a comparatively new concept in on-shore geotechnical engineering. The first on-shore application was via a device called ENPASOL in the early 1970s [1]. It is a system that is capable of recording various drilling parameters, such as bit torque, bit downthrust and drilling speed. It is typically used on rotary destructive drilling rigs and can sometimes be of use on coring rigs. In the latter case, the main use is to complete the core description in case of poor recovery.

The aim of instrumented drilling is to capitalize on the "fine" data, which is available whenever holes are drilled. In tunneling projects, for example, it has become common to install grout tubes, 'Tube-a-Manchettes', during the preconstruction phase of the project, so that grout injections can later be conducted to suppress settlements caused by ground loss. The drilling parameters obtained from the grout holes may then be used either to confirm local stratigraphy or to determine the appropriate grout to be used [2,3]. As a result, the method has also been deployed in continental Europe specifically as a ground investigation tool, especially for soil improvement projects $[4,5]$.

An instrumented drilling machine uses a set of pressure transducers placed at various locations of the hydraulic circuits of the machine in order to measure mud pressure, torque, downthrust and holdback. The measurements are affected by machine vibrations, which contribute largely to the random content of drilling signals. Proper filtering of the noise perturbations, correcting data corrupted by faulty equipment, and compensating for any environmental effects such as temperature and humidity are required to extract best information from the raw signal for local soil characterisation. It may also be possible to interpret the noise and

*Address correspondence to this author at the Department of Civil Engineering (NTUT2656), National Taipei University of Technology, No 1, Sec 3, ZhongXiao E. Rd., Taipei 106, Taiwan; Tel: +886 955154891; Fax: +886 227814518; E-mail: mwgui@ntut.edu.tw characterise the machine vibrations since they can be related to the interaction between the soil and the drilling bit.

The aim of this paper is to explore the use of standard signal processing techniques to assess useful signals obscured by random noise. Firstly, we will briefly describe the background of the two field tests conducted in central London. Secondly, we will show how spectral analysis and autocorrelation can be used to detect random noise in a signal. Thirdly, after quantifying the noise, we will explore the use of three digital filters to reduce the noise in the raw signal. A parametric study will also be performed to find out the corresponding filtering weight or order for each of the drilling parameters. Finally, cross-correlation will be used to demonstrate the repeatability and reliability of the drilling data obtained from the two sites. It is not the intention of this paper to show the interpretation of the instrumented drilling data; this topic has been dealt with in $[6,7]$.

\section{FIELD TESTS}

Field tests had been performed at two locations in London (Site A and B). For the purpose of ground investigation work six instrumented drilling tests have been conducted on Site A. All the tests were conducted using a hydraulically operated and crawler mounted rotary drilling rig and they were vertically drilled to a depth of $30 \mathrm{~m}$. Drilling rods used were $90 \mathrm{~mm}$ diameter API rods with a $101 \mathrm{~mm}$ diameter blade and/or tricone bit. To record drilling parameters the drilling rig was equipped with devices such as pressure transducers and a movement transmitter sensor.

For Site B, thousands of 'tubes-a-manchettes' holes were drilled for grouting purpose. Out of these, a total of 300 holes were drilled using the instrumented borehole drilling machine as the grouting contractor had decided to design their grout properties based directly on the drilling information. These holes were drilled using different types of machine (e.g. KLEMM 804 and SM 305). The drilling depth was about $15 \mathrm{~m}$ for this site.

Drilling method affects the drilling process and the quality of the data; hence, some of the drilling operation was standardized. For Site A, all the drilling were conducted un- 
der a constant drilling fluid flow rate, a constant drill head rotational speed of $120 \mathrm{rpm}$, a constant net downthrust, and a constant drilling speed; hence the test at Site A is defined here as standardized test. For Site B, the drilling fluid flow rate, the drill head rotational speed, the net downthrust and the drilling speed were inconsistent from hole to hole; hence, the test in Site B is defined here as non-standardized test.

The drilling parameters recorded were: depth of drilling, drilling fluid or mud pressure, torque, downthrust, holdback pressure, drilling speed, rotation speed and time to drill 5 $\mathrm{mm}$. The holdback pressure was discounted from the total downthrust to obtain the net or effective downthrust. Analogue signals from some of these instruments were converted into digital signals via a junction box. The digital signals were then stored in a real time drilling data acquisition system that was attached to the drilling rig. The stored data can then be retrieved for further processing. A typical output obtained from the instrumented drilling is plotted in Fig. (1), annotated with regard to the stratigraphy revealed in cores. The raw data have obviously been affected by noise, represented by many spikes in Fig. (1). In general, the following observations can be made:

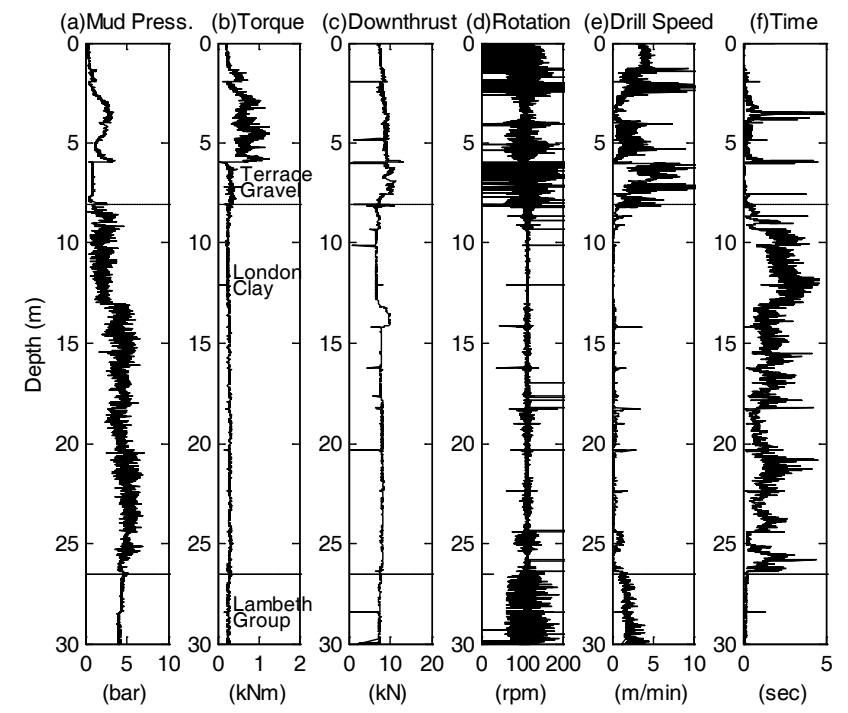

Fig. (1). A typical instrumented borehole drilling 'raw' data (first 8 $\mathrm{m}$ was drilled with casing; soil layers were determined from rotary coring samples).

(1) Mud pump pressure in the sand and gravel layer (Terrace Gravel) was less noisy and has a lower average value than in the clay layer (London clay), Fig. 1(a).

(2) A higher and noisier torque was more likely to be seen in the sand and gravel layer than in the clay layer, Fig. 1(b).

(3) A reasonably constant downthrust had been provided, as shown in Fig. (1(c)). The spikes in the signal are related to the rod change.

(4) Likewise, Fig. 1(d) also shows that a relatively constant rotation speed was also provided, although the data was severely affected by noise, especially in the Terrace gravel layer and the Lambeth group (mixed layers of sands and clays).
(5) A higher drilling speed was more likely to be encountered in the sand and gravel layer than the clay layer, Fig. 1(e). Noisy data can be seen in the sand and gravel.

(6) Time to drill $5 \mathrm{~mm}$, Fig. 1(f), was the reciprocal of the average drilling speed over $5 \mathrm{~mm}$; the longer the drilling time, the harder the formation being drilled. Here, noisy data could also be seen in the clay layer.

\section{NOISE DETECTION FOR FIELD DATA}

Although it seemed that the observed noise coming from machine vibrations could be used to correlate with the ground properties, it was decided in this study to eliminate the random noise from the raw data as the first step of data interpretation. Because the drilling data were recorded by the data acquisition system without any analogue filter, we adopted digital filtering to separate the noise from the signal. In signal processing, there are two common methods to detect noise in a signal: spectral analysis and auto-correlation analysis.

\section{Spectral Analysis}

One useful procedure to detect noise is to compute the spectral density, which can be obtained by performing Fourier transforms on a particular signal. The essential concept of the Fourier technique is that a periodic function can be broken down into its harmonic components and that a periodic function may be synthesized by adding together its harmonic components. The spectral density is simply a measure of how much energy is contained within its harmonic components. Estimation of spectral density is useful in a variety of applications; the concept of the power spectral density function is central to the random vibration theory that has been used for many years in the study of dynamic systems. It has also been used extensively in the detection of signals buried in wide-band noise [8].

Spectral density can be computed as amplitude or power (taken as the square of the amplitude). The amplitude spectrum was chosen for this study. The amplitude spectrum for each drilling signal was calculated using Matlab ${ }^{\circledR}$ [9]. The transformation length was taken to be the data length even though it was recommended that a transformation length with a power of 2 should be adopted for fast execution [10]. In order to eliminate the false ripple in the spectrum, a 'Hanning' window was employed and its length was set to be equal to the length of the Fourier transform [8]. According to [8], the effect of windowing in the frequency domain is that the spectrum is only usefully available between zero frequency and half the sampling frequency (Nyquist frequency).

Signals and the buried noise are normally recorded with respect to time and a Fourier transform calculates the spectral density of the signal and presents it in the frequency domain where the unit is Hertz (Hz). However, the drilling signals were recorded at a constant depth interval of $5 \mathrm{~mm}$, which implied that the time interval required to register each signal was not uniform. It might be possible to do some interpolation to obtain a uniform time interval but this is not so advisable as interpolation might give us misleading results. Hence, in the following analysis, we shall perform the Fourier transform analysis with respect to depth. The unit of the frequency is $1 /$ meter $\left(\mathrm{m}^{-1}\right)$ with a sampling frequency of 
$1 / 0.005=200 \mathrm{~m}^{-1}$. For a data record of $30 \mathrm{~m}$ drilling, there were therefore 6000 points sampled. It must be pointed out that the sampling frequency does not affect the estimation of the spectral density, it was only used to scale the frequency axis $\left(\right.$ Nyquist $\left.=100 \mathrm{~m}^{-1}\right)$ for the graph plotting.

Borehole drilling data taken from Sites A and B were analyzed using spectral analysis. The amplitude spectrum for each drilling parameters (mud pressure, torque, thrust, rotation, speed, holdback pressure, and time) are plotted in Fig. (2) for one of the Site A data. In general, all plots look similar to the amplitude spectrum density (ASD) plot for white noise with no discernible peak, except in the low frequency range. This indicates that the drilling signals mainly consist of low frequency harmonic components and white noise. Severe noise was recorded in the rotation speed signal (Fig. 2(e)), where there was no obvious peak (harmonic component) at all throughout the whole frequency range.

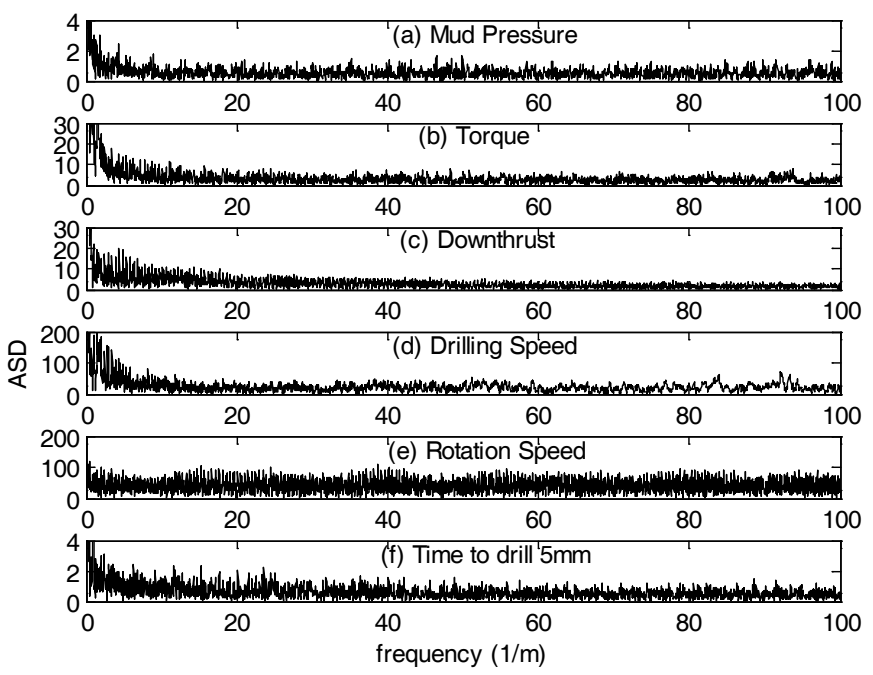

Fig. (2). Amplitude spectrum density for each raw drilling parameter.

\section{Auto-Correlation}

The second technique that is also commonly used to detect noise is auto-correlation analysis. The auto-correlation function arises when a signal is correlated with itself. It is useful to identify time-scales within a signal where repetitive appearance of a pattern or feature occurs. It may be more effective for revealing the presence of wide-band periodic signals that have less distinctive waveforms. If the signal is defined as a finite energy sequence $x[n]$, Eqn. (1) below represents the auto-correlation function $(\mathrm{ACF})$ :

$$
r_{x x}[k]=\sum_{n=-\infty}^{\infty} x[n] x[n+k]
$$

where the sequence is compared with the same sequence that is advanced by $k$ units. The values of $k$ are often referred to as lags.

Auto-correlation was also performed on the drilling parameters taken from sites A and B. Each of the ACF quantities, normalized against its maximum value, is plotted against 'lag' (sample spacing) in Fig. (3) for one of the Site A data. Since the depth data were equally spaced, we could infer depth, if desired, from the 'lag' axis (depth = 'lag' $\mathrm{x}$
$0.005 \mathrm{~m})$. A triangular shape shows that when the lag increases (meaning two depth instants further apart), the two quantities remain perfectly correlated. The reason was that a constant signal of given duration (here, 6000 points) was regarded as being preceded and succeeded by zeros. When it was multiplied by the same signal with a lag, some of the extreme zeros cancel the original signal. This occurred increasingly until the lag equals to the signal length. The plot towards the left and right hand ends was never be so accurate because the number of data points that was actually used to compute the correlation for large lags was significantly less than when the lag was small. When the lag was zero, the auto-correlation was a maximum and just gives the variance in the reading. This makes physical sense since we were trying to correlate two quantities that have the same exact value all the time, i.e. perfectly correlated. Also, the correlation plot should be symmetrical about the origin if the data was real valued (not complex numbers).

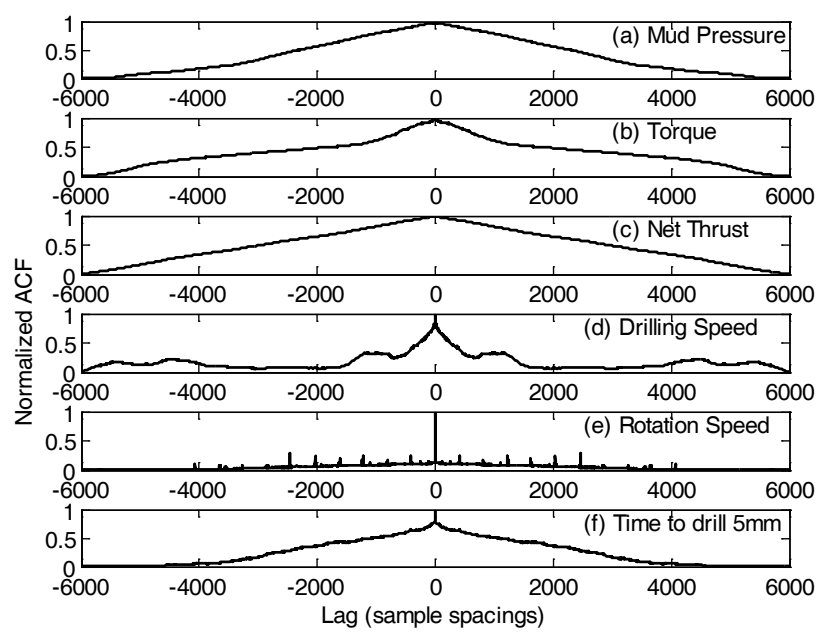

Fig. (3). Normalized auto-correlation function for each raw drilling parameter.

In terms of borehole drilling data, a broad triangle like Fig. (3(c)) would indicate that the downthrust has a reasonably constant value throughout the test since it was intended to be kept constant during the test. Localized effects, around the crest and towards the ends of the ACF triangles, have been observed in the torque parameter (Fig. 3(b)). This was caused by different torque values in the Terrace Gravel layer, London Clay layer and Lambeth Group layers. The localization effects were even greater in the drilling speed parameter, Fig. 3(d), where the ACF curve decreased quickly from 1 to 0.08 , remained constant for about $12 \mathrm{~m}$, and then increased again to 0.2 before falling to zero. The localization effects indicated that there were 3 discernible ranges of drilling speed throughout the depth, as confirmed in Fig. (1(e)), where the average speed was $2.9 \mathrm{~m} / \mathrm{min}$ in the Terrace Gravel layer, $0.25 \mathrm{~m} / \mathrm{min}$ in the London Clay layer, and $2.2 \mathrm{~m} /$ min in the Lambeth Group layer. The mud pressure parameter also has a localization effect but not as clearly defined as the torque and drilling speed parameters. The parameters with localization effect can be used to identify soil layers.

The sharp spike at zero lag in drilling speed, rotation speed and time, Fig. 3(d) to (f), reveal that these data are affected by a lot of noise. The very sharp spike at zero lag 
together with low normalized ACF value means that the rotation speed was severely affected by white noise, showing a very weak self-correlation. Perhaps, no significant statistical correlation should be expected between the rotation speed parameter and the shifted version of itself, Fig. 3(e). The rotation speed was kept constant but the small sharp spikes that coincided with the rod changes distorted the ACF value. This was represented by the normalized ACF that remains at unity for zero lag, and becomes much smaller elsewhere.

\section{FILTERING OF FIELD DATA}

Since all the drilling data reported were obtained using systems that did not have a built-in analogue filter, digital filters were adopted here to filter the noise from the raw data. The advantages of digital filters are: (i) they are software programmable, (ii) they are stable and predictable, (iii) they do not drift with temperature or humidity, and (iv) they have superior performance-to-cost ratio. Signal processing toolboxes, which come with software such as Matlab, are all capable of performing this task. There are two types of digital filter: one that is used to remove noise in the time-domain and one that is used to remove noise in the frequencydomain.

\section{Moving Average Filter}

A moving average filter can be used to remove noise from a signal in time-domain. In particular, high-frequency noise in a signal can be reduced by running a moving average filter [11]. Consider a set of points in discrete time that represents a straight line. The smoothed $i$-th value of the signal is computed from the original noisy signal $x$ as an average of neighboring values around $i$. The averaging of neighbors around the $i$-th discrete element gives the value of $x_{i}$ exactly. If random noise is added to the initial points, the averaging operation tends to cancel the noise [11]. The forward moving average equation is given by:

$$
y_{i}=\sum_{n=-p}^{0} \kappa_{n} \cdot x_{i+n}
$$

where $p$ is an integer number representing a filtering weight and $\kappa$ is defined as the 'kernel' that contains the weights to be applied to neighboring elements. The sum of all weights in a smoothing kernel must be equal to one $\left(\Sigma \kappa_{n}=1\right)$. In theory, we can define $\kappa_{i} \neq \kappa_{i+1} \neq \kappa_{i+2}$, and so on, however, for simplicity we will only use a simple moving average filter with $\kappa_{\mathrm{i}}=\kappa_{\mathrm{i}+1}=\kappa_{\mathrm{i}+2}=1 / p$ in the following analysis.

It is vital to have a zero-phase filtering for all kinds of digital filter. This can be done by processing the input data in both forward and reverse directions. After filtering in the forward direction, it reverses the filtered sequence and runs it back through the filter. Hence, the reverse moving average is now represented by:

$$
y_{i}=\sum_{n=0}^{+p} \kappa_{n} \cdot x_{i+n}
$$

The resulting sequence has precisely zero-phase distortion and double the filter order.

For parametric study, a moving average filter with filtering weight, $p$, of 5, 10 and 20 were selected to filter the time parameter of the drilling data (Fig. 4(a) to (c)). The corresponding kernel values, $\kappa$, are thus $1 / 5,1 / 10$ and $1 / 20$. It can be seen that as $p$ increases, the data become smoother, Fig. 4(d) to (f). The selection of $p$ and hence, $\kappa$ was critical for noise control. If $p$ was very big (long), smoothing would remove significant low frequency components, Fig. 4(c), which might be relevant to the signal; if $p$ was too small (short), only the highest frequency noise would be removed and there was a possibility that some noise would still remain, Fig. 4(a).
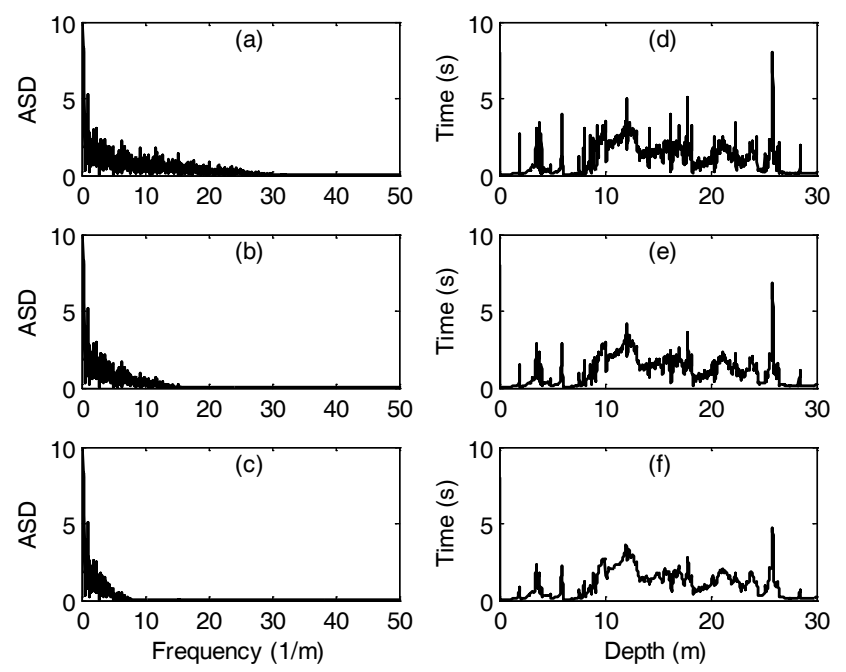

Fig. (4). Moving average filter with filtering weight p: (a) 5; (b) 10; (c) 20; and (d)-(f) the corresponding filtered value for time.

\section{Median Filter}

A median filter can also be used to filter noise in the time-domain. The median, $y_{i}$, is essentially the 'middle' value of a set of data values when they are sorted in ascending or descending order. Matlab applies an order $k$, onedimensional median filter to the signal. When $k$ is odd, $y_{i}$ is the median of $x_{[i-(k-1) / 2]}, \ldots, x_{[i+(k-1) / 2]}$. On the other hand, when $k$ is even, $y_{i}$ is the median of $x_{[i-k / 2]}, x_{[i-k / 2+1]}, \ldots, x_{[i+k / 2-1]}$. Hence, in both cases, the median does not use all the data values in its calculation. When the filtering order, $k$, is odd it only uses one data value (the middle one); when $k$ is even it uses the two middle values. The median is thus, unlike the mean or average value, a relatively stable measure and is not affected by the extreme values.

For a comparison with the moving average filter, a median filter with filtering orders of 5, 10 and 20 was used to process the time parameter data (Fig. 5(a) to (c)). The corresponding filtered results are presented in Fig. $\mathbf{5}(\mathbf{d})$ to $(\mathbf{f})$. Again, it can be seen that the higher the filtering order the smoother the result is.

\section{Butterworth Filter}

A Butterworth filter is generally used to control noise in frequency-domain. There are three classical types of Butterworth filter: low-pass, high-pass and band-pass. A low-pass filter keeps frequency components below the cut-off frequency while frequency components above the cut-off fre- 
quency are rejected. It is usually used in suppressing highfrequency noise. A high-pass filter lets the high-frequency components "pass" while the frequencies below the cut-off frequency are rejected. It is used to remove the static component or zero-frequency offset in a signal. The combination of low- and high-pass filters is a band-pass filter. It is used to preserve a certain threshold of frequency while nullify others. It seems that the drilling data may be represented by low frequency components (Fig. 2); therefore, the most suitable filter to be used is the low-pass filter.
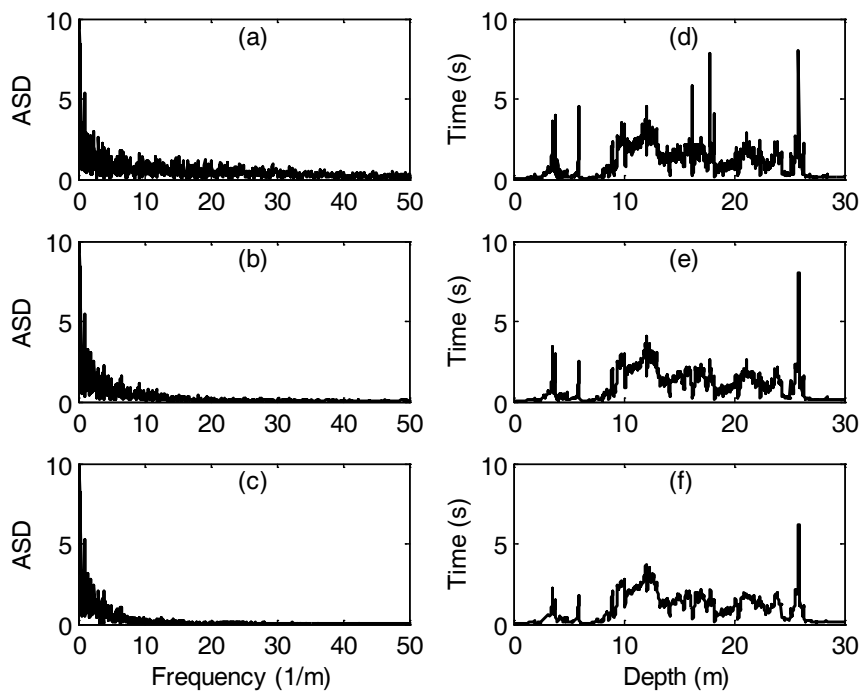

Fig. (5). Median filter with filtering order $k$ : (a) 5; (b) 10; (c) 20; and (d)-(f) the corresponding filtered value for time.

Here, a $3^{\text {rd }}$ order low-pass Butterworth digital filter was used to filter the noise from the drilling raw signals. Cut-off frequencies of 20/100, 10/100 and 5/100 $\mathrm{m}^{-1}$ were chosen to filter the time parameter (Fig. 6(a) to (c)). The cut-off frequency must be a number between 0 and 1 , where 1 corresponds to half the sampling frequency. The Butterworth results are plotted in (Fig. 6(d) to (f)) and it shows that the higher the cut-off frequency the more the original data (which also includes low frequency noise) would be preserved.
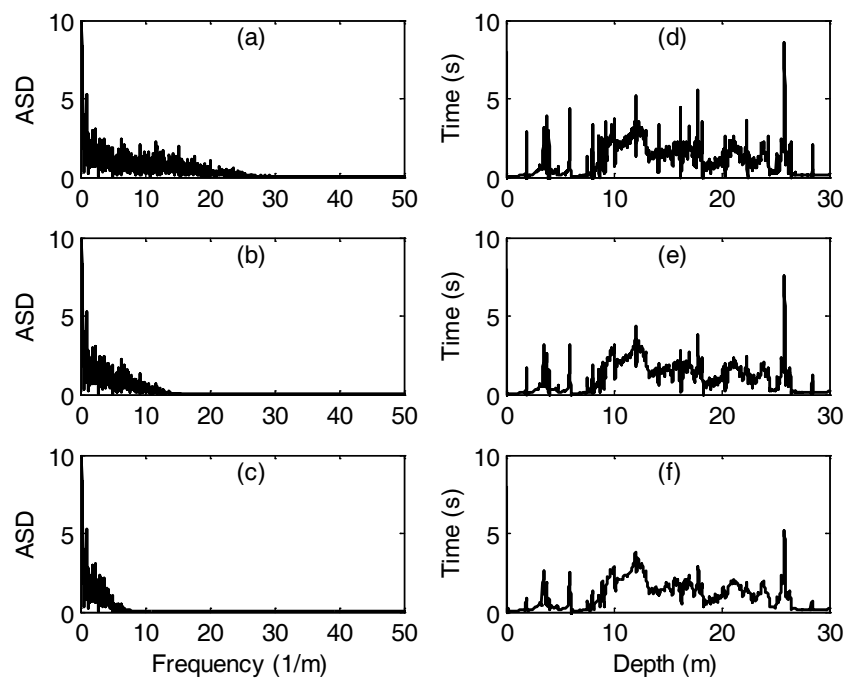

Fig. (6). Butterworth filter with cut-off frequency: (a) 20; (b) 10; (c) 5; and (d)-(f) the corresponding filtered value for time.
Fig. 7(a) shows the comparison of the time data after applying the moving average filter $(\mathrm{p}=20)$ and median filter $(k$ $=20$ ), while Fig. 7(b) shows the comparison of the time data after applying the median filter $(k=20)$ and Butterworth filter (cut-off frequency $=5 / 100$ ). In general, it can be seen that the median filter was capable of getting rid of more 'spikes' than the moving average and the Butterworth filters. However, the median filter did not use all the available information contained in the original data during filtering, which might be unwelcome in certain situations, but this was good for our drilling data, which contains sharp spikes.
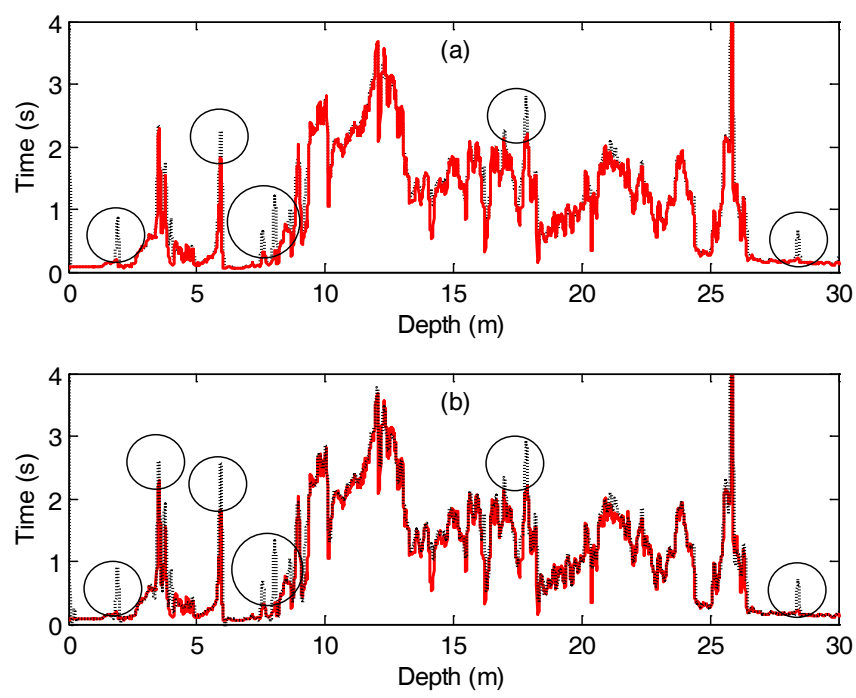

Fig. (7). Comparison of time data filtered by: (a) moving average filter with $\mathrm{p}=20$ (black dashed-line) and median filter with $k=20$ (red solid-line); (b) Butterworth filter with cut-off frequency of $5 / 100$ (black dashed-line) and median filter with $k=20$ (red solidline). Black circles denote the 'spikes' left in the data filtered by (a) moving average filter, and (b) Butterworth filter.

\section{Which Filters}

It is hard to recommend a unique filter together with its filtering weight (moving average), order (median) or cut-off frequency (Butterworth) for each of the drilling signal. A parametric study, to find out the weight, order and cut-off frequency for each of the above-mentioned filter was thus carried out. Noise is deemed to be completely filtered when the spike in the normalized ACF plot, Fig. (3), at zero lag is diminished. A typical result, using the median filter on each of the drilling parameters together with the corresponding normalized ACF, are presented in Fig. (8). The disappearance of the original sharp spike in the drilling speed, rotation speed and time signals in the normalized ACF plots shows that the noise in these signals was substantially removed, in particular for the rotation speed. As for mud pressure, torque and downthrust, the filtering order was determined after most of the spikes disappeared from the original signal.

This exercise was performed for the entire Site A tests and a global value of the filtering weight, order and cut-off frequency for each of the drilling parameters is derived. They are summarized in Table 1. A low filtering weight or order but high cut-off frequency reveals that signals for mud pressure, torque, down-thrust and holdback contain less noise than the drilling speed, rotation speed and time signals. It seems that the outputs of the instrumentation for mud pres- 

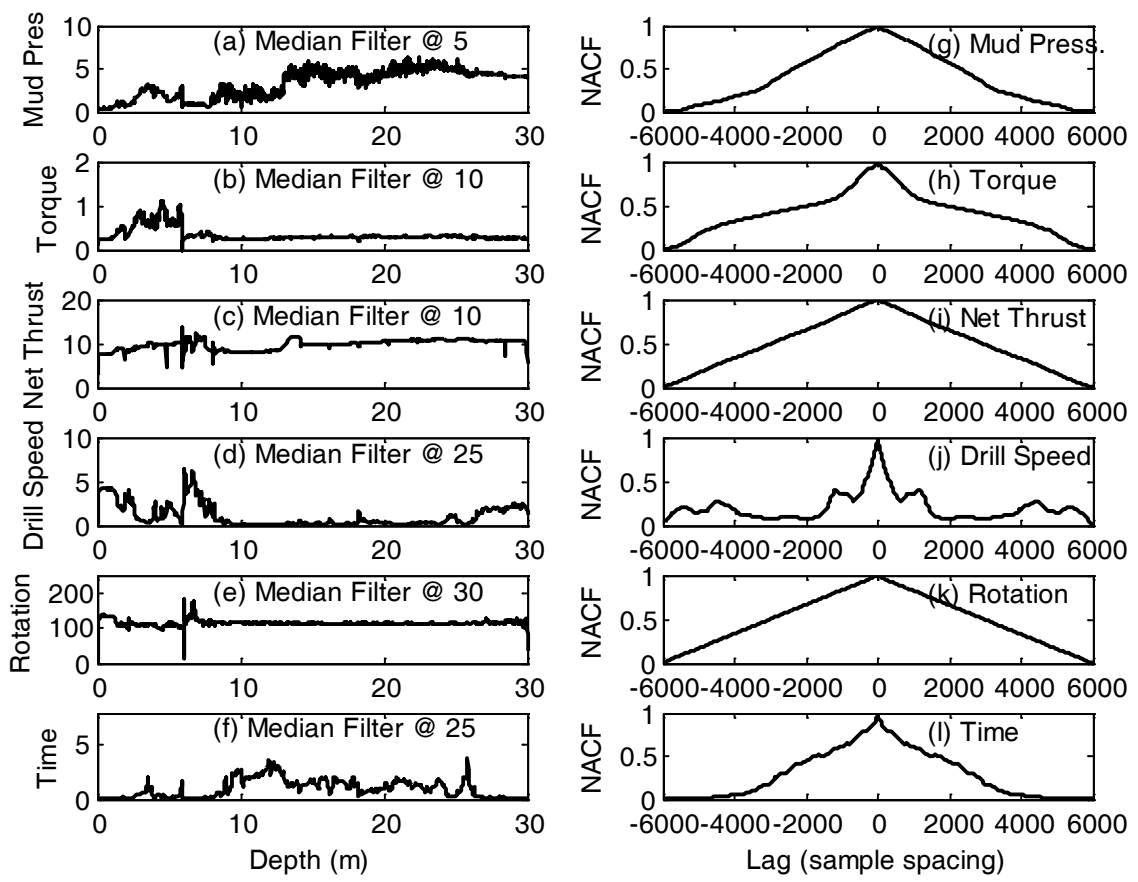

Fig. (8). Determination of filtering order of median filter for drilling parameter and their corresponding normalized auto-correlation function (NACF).

Table 1. Filter Weight, Order and Cut-off Frequency

\begin{tabular}{|c|c|c|c|}
\hline Drilling Parameters & Weight of Moving Average Filter $(\boldsymbol{p})$ & Order of Median Filter $(\boldsymbol{k})$ & Cut-off Frequency of Butterworth Filter \\
\hline \hline Mud pressure & 5 & 5 & $10 / 100$ \\
\hline Torque & 10 & 10 & $10 / 100$ \\
\hline Thrust & 10 & 10 & $10 / 100$ \\
\hline Hold-back & 10 & 25 & $2 / 100$ \\
\hline Drilling speed & 25 & 30 & $2 / 100$ \\
\hline Rotation speed & 30 & 25 & $2 / 100$ \\
\hline Time to drill 5mm & 25 & & \\
\hline
\end{tabular}

sure, torque, downthrust, and holdback are less sensitive to the machine/environmental noise.

\section{CHECK OF TEST REPEATABILITY USING CROSS- CORRELATION}

Instrumented drilling is a relatively new ground investigation tool; therefore, it is vital to check the repeatability of the drilling data. There may be several ways to check the repeatability of a test. Perhaps, the most direct way is to perform a few tests at a homogeneous site and then compare the test data, while the indirect way is to use the crosscorrelation function. Cross-correlation function (CCF) is capable of indicating similarity of two signals as a function of the delay between them. Here, the CCF was used to check the similarity of the drilling data obtained from sites $\mathrm{A}$ and B. As mentioned earlier, tests performed at Site A were standardized test because these tests were performed using the same testing procedures, instrumentation and equipment. On the other hand, tests performed at Site B were nonstandardized because they were performed using unspecified by generically similar testing procedures, different instrumentation and equipment.

The cross-correlation of two signal sequences $x[n]$ and $y[n]$ is a third sequence $r_{x y}[k]$, defined as:

$$
r_{x y}[k]=\sum_{n=-\infty}^{\infty} x[n] y[n-k]
$$

where the second sequence $y[n]$ is delayed by $k$ units relative to the first sequence $x[n]$, and the sum of the product terms is then evaluated. This is done for all values of $k$. To obtain a better comparison, the cross-correlation result is normalized against the auto-correlation values (Eq. 1) of both signals being compared as follow: 


$$
\frac{r_{x y}[k]}{\sqrt{r_{x x}[k]^{2}+r_{y y}[k]^{2}}}
$$

where subscript $x$ is a drilling parameter and subscript $y$ is the same drilling parameter but from the other test. For example, the torque parameter of test A1 was compared with the torque parameter of test A2, A1 with A4, etc. A good cross-correlation of any two tests is defined by a triangular shape of CCF profile and a close to unity CCF value at zero lag.

\section{Standardised Test at Site A}

Cross-correlation was performed for each of the drilling parameters of tests A1, A2 and A4 in Fig. (9). Qualitatively, the overlapping or near-overlapping of CCF profiles in mud pressure and torque, Figs. 9(a) and (b), show that there was some consistency in the quality of the data between each of these standardized tests. From the quantitative point of view, the normalized cross-correlation value for mud pressure, torque and net thrust was about 0.7 , which meant that these results were still not perfectly correlated. However, there was an even poorer correlation between the drilling speed data, Figs. 9(d), which only has a maximum normalized CCF value of about 0.4 . In this case, the data might not be so consistent.

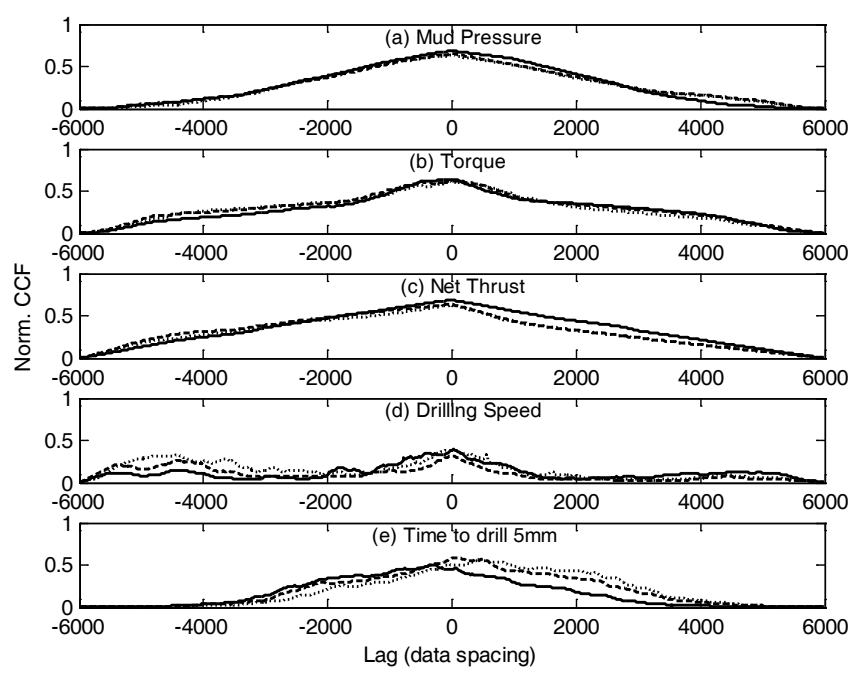

Fig. (9). Cross-correlation function to examine the similarity of Site A data. Solid-line: correlation between A1 and A2; dashed-line: between $\mathrm{A} 1$ and $\mathrm{A} 3$; dotted-line: between $\mathrm{A} 2$ and $\mathrm{A} 4$.

\section{Non-Standardised Test at Site B}

The drilling tests performed at Site B were nonstandardized ones. The machine, the instrumentation and the drilling procedures were not standardized like the Site A test. The quality of these non-standardized data could be compared using the cross-correlation function. Three vertically drilled tests, B1, B2 and B3, which were only a few meters away from each other, were randomly selected for this analysis. If these were standardized tests, a high degree of similarity between these tests would have been expected, as for the Site A data. However, from their normalized CCF results plotted in (Fig. 10), except for the torque in (Fig. 10(b)), the CCF profiles for each of the drilling parameters did not overlap each other, i.e. inconsistencies exist in the mud pressure, drilling speed and time parameters. The nonsymmetrical envelope for the net thrust, Fig. 10(c), revealed that it was not controlled properly. The non-overlapping and non-symmetrical envelopes were even more severe for the drilling speed and time parameters, Figs. 10(d) and (e). These data were thus unreliable than they needed be. The unreliability and inconsistency must be caused by the nonstandardized method of drilling.

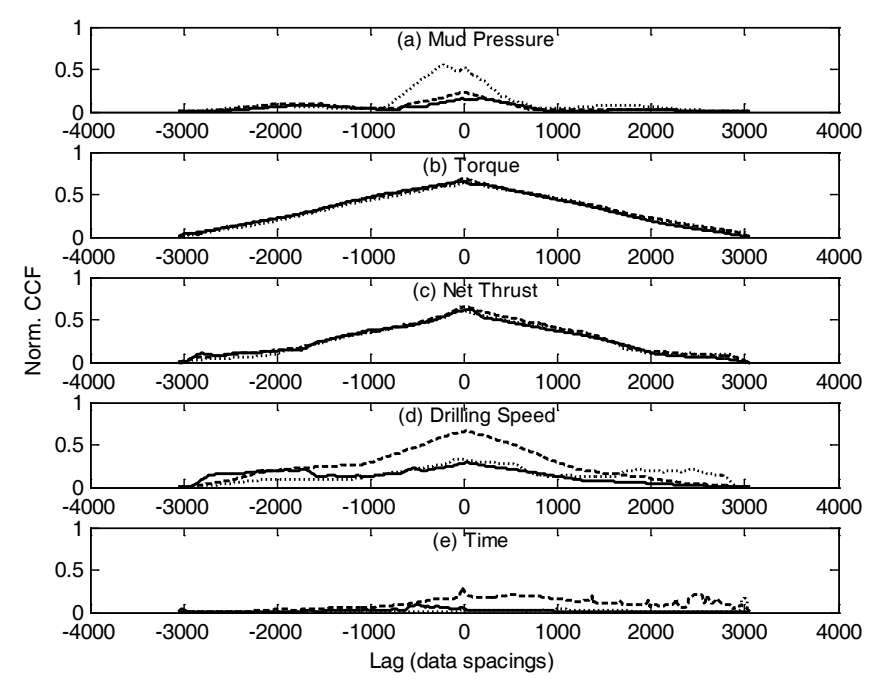

Fig. (10). Cross-correlation function to examine the similarity of Site B data. Solid-line: correlation between B1 and B2; dashed-line: between B1 and B3; dotted-line: between B2 and B3.

\section{CONCLUSIONS}

The aims of this study were to check the consistency of the data and to detect and remove the noise buried in drilling data. Signal processing techniques, spectral analysis and auto-correlation were used to detect the existence of unwanted noise in the data. The analysis shows that most of the drilling data were affected by noise; the drilling speed, rotation speed and time data seem to contain more noise than the other parameters. Fortunately, the noise could be removed by using an appropriate digital filter. The use of time-domain filters such as the moving average and median filters were deemed to be adequate for this purpose; and its filtering weight, order or cut-off frequency could also be determined from the auto-correlation analysis. Using cross-correlation, it was found that the results obtained from the standardized tests were more consistent than the non-standardized tests. This shows that proper standardization needs to be adopted for any instrumented drilling data to be useful for subsequent data interpretation.

\section{ACKNOWLEDGEMENTS}

The author is grateful to the assistance provided by Prof M D Bolton and Prof K Soga of CUED and J P Hamelin of Soletanche Bachy. 


\section{REFERENCES}

[1] B. S. Chun, Y. S. Chae, B. H. Chung and H. S. Choi, "A case study of system grouting using 'ENPASOL' \& 'SINNUS'," Proc. 9th International Offshore and Polar Engineering Conference, France, 1999, pp. 608-612.

[2] P. Denbigh, System Analysis \& Signal Processing. Addison-Wesley Longman Ltd., 1998.

[3] M. W. Gui, K. Soga, M. D. Bolton and J. P. Hamelin, "Instrumented borehole drilling for subsurface investigation," Journal of Geotechnical and Geoenvironmental Engineering, ASCE, vol. 128(4), pp. 283-291, 2002.

[4] M. W. Gui and J. P. Hamelin, "Development of an instrumented borehole drilling system for ground investigation," Electronic Journal of Geotechnical Engineering, vol. 9(A), pp. 350, 2004. [Online]. Available: www.ejge.com [Accessed 2004]
[5] H. Guillaud and J. P. Hamelin, "Computerized grouting design and control," Proc. International Symposium on Prediction versus Performance in Geotechnical Engineering, Bangkok, 1992.

[6] J. P. Hamelin, J. Levallois and P. Pfister, "Enregistrement des parametres de forage: nouveaux developments," Bulletin de l'Association Internationale de Geologie de l'Ingenieur, No. 26, pp. 83-88, 1982.

[7] Matlab Version 7 User's Guide. The Math Works, Inc., Natick, 2005.

[8] D. E. Newland, An Introduction to Random Vibrations, Spectral \& Wavelet Analysis, Longman Publ., 1994.

[9] A. Pazuki and S. R. Doran, "Soil Investigation for cross passages," Proc. XI European Conference on Soil Mechanics and Foundation Engineering, 1995.

[10] P. Pfister, "Recording drilling parameters in ground engineering," Journal of Ground Engineering, vol. 18(3), pp. 16-21, 1985.

[11] J. C. Santamarina and D. Fratta, Introduction to Discrete Signals and Inverse Problems in Civil Engineering, ASCE Press, 1998.

(C) Meen-Wah Gui; Licensee Bentham Open.

This is an open access article licensed under the terms of the Creative Commons Attribution Non-Commercial License (http://creativecommons.org/licenses/by$\mathrm{nc} / 3.0 /$ ) which permits unrestricted, non-commercial use, distribution and reproduction in any medium, provided the work is properly cited. 